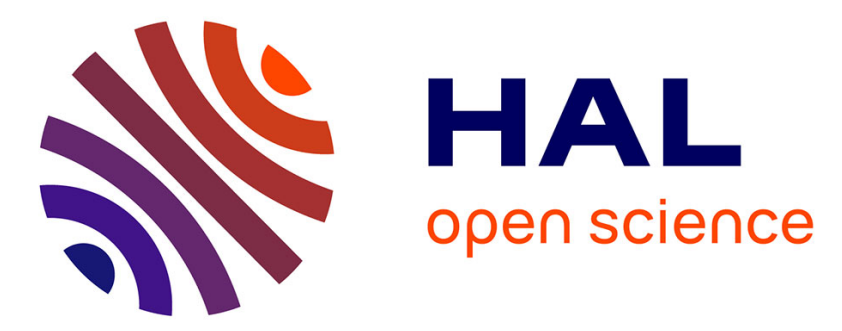

\title{
Some advances towards a better understanding of wave propagation in civil engineering multi-wire strands
}

\author{
Laurent Laguerre, Fabien Treyssede
}

\section{To cite this version:}

Laurent Laguerre, Fabien Treyssede. Some advances towards a better understanding of wave propagation in civil engineering multi-wire strands. ULTRASONIC WAVE PROPAGATION IN NON HOMOGENEOUS MEDIA, 2009, 128, pp 123-135. 10.1007/978-3-540-89105-5_11 . hal-00918402

\section{HAL Id: hal-00918402 \\ https://hal.science/hal-00918402}

Submitted on 13 Dec 2013

HAL is a multi-disciplinary open access archive for the deposit and dissemination of scientific research documents, whether they are published or not. The documents may come from teaching and research institutions in France or abroad, or from public or private research centers.
L'archive ouverte pluridisciplinaire HAL, est destinée au dépôt et à la diffusion de documents scientifiques de niveau recherche, publiés ou non, émanant des établissements d'enseignement et de recherche français ou étrangers, des laboratoires publics ou privés. 


\title{
Some advances towards a better understanding of wave propagation in civil engineering multi-wire strands
}

\author{
Laurent Laguerre $^{1}$ and Fabien Treyssede ${ }^{2}$
}

\begin{abstract}
Steel members of civil engineering structures undergo degradations mainly due to corrosion and mechanical fatigue. In this context, non-destructive inspection techniques using mechanical guided waves have potential to monitor these structures. Even if wave propagation is not yet fully understood in these structures, useful results can be derived for inspection methodology by using wave modeling in cylindrical waveguide, embedded or not. However, further improvement can be expected from the development of wave propagation simulation tools for real-life structures. Indeed, several difficulties arise in the understanding of guided ultrasonic waves in such structures, partly due to the helical geometry and the inter-wire coupling effects. Moreover, these structures are pre-stressed and can be free or embedded in solid material. This paper shows some recent research results at LCPC. A first part deals with experimental results on the guided wave propagation in a commonly used steel member, the seven wire strand (i.e one straight single cylindrical wire surrounded by six helical wires). The second part aims at numerically investigating the propagation of elastic waves in free helical waveguides. A numerical method is chosen based on a semi-analytical finite element technique that relies on a specific non-orthogonal curvilinear coordinate system. This system is shown to be translationally invariant along the helix centerline so that a spatial Fourier transform can be explicitly performed along the axis to reduce the problem to two dimensions. The method can thus readily be used for the analysis of helical structures by considering the special case of no curvature. Results for single straight and helical wires are first computed. A dispersion analysis for a seven wire strand with simplified contact conditions is then performed.
\end{abstract}

\footnotetext{
${ }^{1}$ Laguerre Laurent

Laboratoire Central des Ponts et Chaussées (LCPC), Centre de Nantes, Division Reconnaissance et Géophysique, Route de Bouaye - BP 4129, 44341 Bouguenais, France, email :Laurent.Laguerre@lcpc.fr

${ }^{2}$ Fabien Treyssede

LCPC, Centre de Nantes, Division Métrologie et Instrumentation, Route de Bouaye - BP 4129, 44341 Bouguenais, France, e-mail :Fabien.Treyssede@lcpc.fr
} 


\section{Introduction}

Multi-wire strands are commonly used as structural members in the field of civil engineering, in bridges constructions (suspended, cable-stayed and prestressed ones) as well as in geotechnics for retaining walls applications. These civil engineering structures suffer from ageing and degradation due to corrosion and fatigue of structural steel members. Guided ultrasonic waves are potentially attractive tools for non destructive assessment of their integrity. They generally combine the ability to inspect, in a single measurement, the whole width of the structure over an appreciable length along the guiding direction. Moreover, they provide a mean to access hidden parts of the structure, such as sections of strand partially or totally grouted in grease or cement. Guided wave non-destructive methodologies of inspection using magnetostrictive or piezoelectric devices are generally proposed, depending on the desired applications.

The goal of this paper is to show some recent advances performed at LCPC dealing with the development of a numerical model for the guided wave propagation in a free seven-wire strand. This common strand configuration is composed of a straight cylindrical wire of $5.4 \mathrm{~mm}$ in diameter surrounded by six helical wires of $5.22 \mathrm{~mm}$ in diameter. These works come within the scope of developing tools to provide a better insight in the interpretation of guided wave propagation in such complicated waveguides for further optimised inspection strategy.

The simplest and classical way to approach the wave propagation in the sevenwire strand is to use the approximation of the infinite cylinder. In particular, experimental studies have been performed in the literature for both a cylindrical bar and a seven wire strand, trying to find analogies between both behaviours that could be interpreted from Pochhammer-Chree solutions, for the bare waveguide case (Kwun et al. 1998, Laguerre et al. 2002, 2003, Lanza di Scalea et al. 2003 and Rizzo and Lanza di Scalea 2004) or the embedded one (Pavlakovic et al. 1999, 2001 and Beard et al. 2003). Even if the non-destructive inspection strategy of a seven-wire strand with guided ultrasonic waves has gained from this approach, the authors point out the limits of Pochhammer-Chree solution for the accurate interpretation of experimental data for the seven wire strand. The theoretical understanding of guided ultrasonic waves in multi-wire strands is still challenging because of the complexity of this structure, i.e. the helical geometry of peripheral wires surrounding the straight inner wire, the inter-wire coupling and contact effects, and the presence of applied loads.

In order to deal with complex geometry, some of the most popular and efficient numerical techniques are based on finite element (FE) methods. The so-called semi-analytical finite element (SAFE) method is a first approach that has been used to study uniform waveguides of arbitrary cross-section - see for instance, (Gavric 1995, Hayashi et al. 2003, Damljanovic and Weaver 2004). (Demma et al. 2005) and (Finnveden and Fraggstedt 2008) investigated toroidal waveguides. 
(Onipede and Dong 1996) extended SAFE methods to study uniformly pretwisted waveguides along a straight axis.

A second approach is based on the theory of wave propagation in periodic structures from Floquet's principle. A review on the topic can be found in Mead 1996. Based on a general theory presented by Mead 1973, some periodic FE approaches and procedures have then been developed - see for instance (Gry and Gontier 1997, Mace et al. 2005).

For modelling a single helical wire, which is a uniform waveguide, both SAFE or periodic FE approaches can be applied. (Treyssede 2007) has proposed a numerical procedure based on a periodic FE approach combined with a specific helical mapping in order to arbitrarily reduce the periodic cell length. (Treyssede 2008) has also recently developed a SAFE method extended to helical waveguides. The weak variational formulation is written in terms of a non-orthogonal curvilinear coordinate system that is translationally invariant along the helix centreline, so that a Fourier transform can be explicitly performed. In this paper, it is shown how this SAFE method can also be readily used to study some more general helical structures, made of both straight and helical wires such as multi-wire strands.

The first part of this paper is devoted to show, from experimental data, the analogy and discrepancies existing between the guided ultrasonic wave propagation into a cylindrical bar and the seven-wire strand in the low frequency regime. This is done through spectrogram analyses of time history signals generated and detected using a longitudinally polarized non-contact magnetostrictive device.

In the second part, one proposes a numerical method allowing the study of elastic guided waves inside helical multi-wire strands. Inter-wire coupling effects are included in the analysis through simplified contact conditions. Prestress and embedment are not considered.

\section{Experimental results}

Magnetostrictive transducers were used herein for the generation and detection of guided ultrasonic waves into steel cylindrical bars and seven-wire strands. The major advantage of non-contact electromagnetic magnetic transducers (EMAT) such as magnetostrictive transducer is in the greater transduction efficiency at low bias fields contrary to Lorentz force based EMATS. Conversely, magnetostrictive EMATS are rather low-frequency transducers (typically $<500 \mathrm{kHz}$ ). These transducers uses the property of ferromagnetic materials to mechanically strain under a variation of their magnetic state (magnetostrictive Joule's effect). The magnetic excitation is though produced by a dynamic electrical current feeding a driving coil. This in turn generated the mechanical wave. The mechanical wave while propagating produces magnetic field variation (magnetostrictive Villari's effet) that can be detected using and inducting coil. Bias magnetic fields are superimposed at the generation and detection to linearize and enhance the transduction. 
The selected bias field longitudinal orientation makes the generation and detection predominantly sensitive to longitudinal guided waves.

In this study, various through-transmissions experimentations were performed for free cylindrical bars and a free seven-wire strand or under applied tensile loads. Time excitation is a gaussian-shaped low radiofrequency burst of bandwidth $\Delta f$ centered at $f_{0}$, and such that $\Delta f / f_{0}=1$ at $-20 \mathrm{~dB}$. Detected guided ultrasonic time waveforms are then studied using a short time Fourier transform (STFT). According to the positioning of transducers (the transmitter and detector locations are at $L / 4$ from the left-end side of the specimen and $L / 2$ respectively, with $L$ the specimen length, see for instance Laguerre et al. 2003), the first wave arrival is the incident wave propagating to the right, the second arrival is the incident wave propagating to the left after one reflection, and the third one is the incident wave propagating to the right after one reflection (and so on for the next arrivals). According to their intrinsic dispersive behaviours in this frequency range, the wavepacket arrivals (Fig. 1) can be identified from Pochhammer-Chree theory as the first and second longitudinal modes $L(0,1)$ and $L(0,2)$ respectively. Similar measurements performed in the same frequency range for a lower diameter show that the waveguide behaviour is limited to the fundamental $L(0,1)$ longitudinal mode, in its weak dispersive region as a consequence of the frequency-to-diameter conservation.
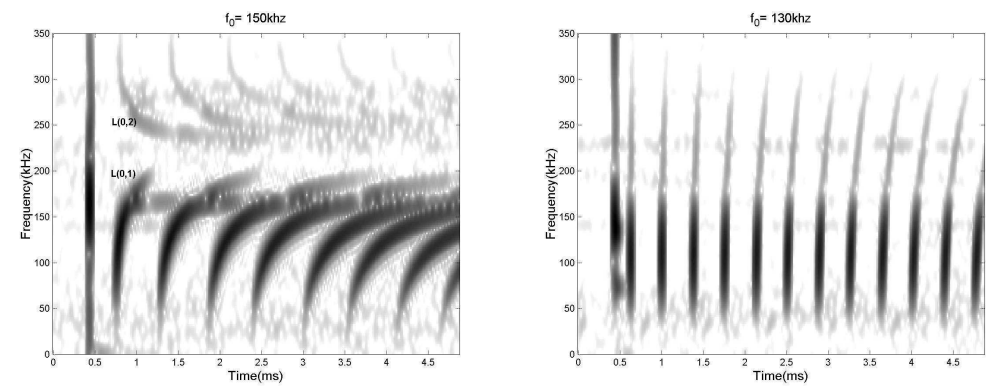

Fig. 1 Fourier spectrograms of magnetostrictive time history signals for (a) a steel cylindrical bar of 6-m length and $15.5 \mathrm{~mm}$ in diameter, and (b) a single central cylindrical wire of 4-m and 5.22 $\mathrm{mm}$ in diameter excited at $\mathrm{f}_{0}=150 \mathrm{kHz}$ and $130 \mathrm{kHz}$ respectively.

These expected above-mentioned results are only quoted here to point out the discrepancies existing between a cylindrical bar and a free seven-wire strand. The Fourier spectrograms associated to the free seven-wire strand are shown in Fig. 2. In the low-frequency region (lower than $50 \mathrm{kHz}$ ), the pulse propagates without significant dispersion, at the bar celerity, while the higher frequency trends cannot be explained at all by the Pochhammer-Chree theory, neither by considering a bar of the same diameter as the strand nor by considering a bar of the same diameter 
as the single central wire. One of the major differences between the cylinder and the strand arises mainly from a frequency band from 60 to $80 \mathrm{kHz}$, electrically excited, but which disappears from the Fourier spectrogram for all wave arrivals.

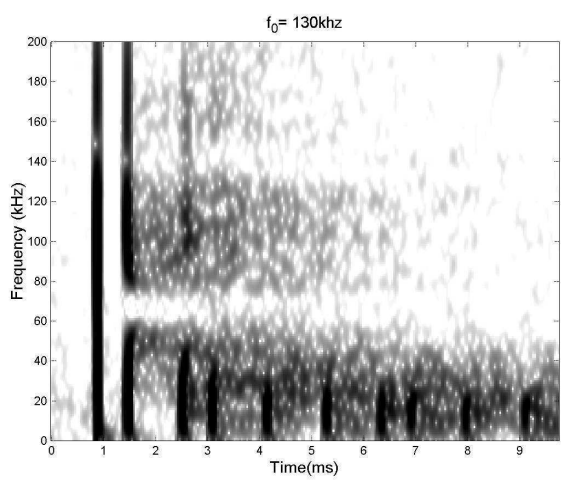

Fig. 2 Fourier spectrograms of magnetostrictive time-history signals for a free seven-wire strand of $10-\mathrm{m}$ length and $15.9 \mathrm{~mm}$ in diameter excited at $\mathrm{f}_{0}=130 \mathrm{kHz}$.

This phenomenon which was first observed by (Kwun et al. 1998) who called it "missing notch-frequency" seems to exhibit a similar pattern as the one of a cutoff frequency. It was confirmed later by (Laguerre et al. 2003, Rizzo and Lanza di Scalea 2004) but still remains unexplained. The "missing notch-frequency" reveals the influence of the seven-wire geometrical structure on the guided wave propagation. Indeed, the origin of this missing notch-frequency can solely be understood, as will be shown in Sect. 3.3, by considering the whole assembling of helical wires around the central cylindrical straight with inter-wire coupling conditions. So this missing notch-frequency phenomenon will be retrieved from the seven-wire strand theoretical propagation model derived in the following section. Moreover, the influence of prestress and inter-wire contacts on the wave propagation is confirmed by the results obtained for a seven wire strand under different tensile loading strengths varying from $2 \%(5.8 \mathrm{kN})$ to $60 \%(141 \mathrm{kN})$ of the breaking load. The non-contact magnetostrictive transducers are particularly well-suited for this loaded strand experimental arrangement since they allow to work on the first wave arrival whose propagation path did not interact with the clamped ends, by positioning them between the anchorages. The Fourier spectrograms Fig. 3 clearly show the modification in the missing notch frequency with load, i.e. an increase in the loading strength leads to the increase in the missing notch frequency (from 60 -to- $80 \mathrm{kHz}$ at $2 \%$ to 80 -to- $100 \mathrm{kHz}$ at $60 \%$ of the breaking load). This demonstrates the influence of prestress and inter-wire contact conditions on guided wave propagation in a seven-wire strand. 


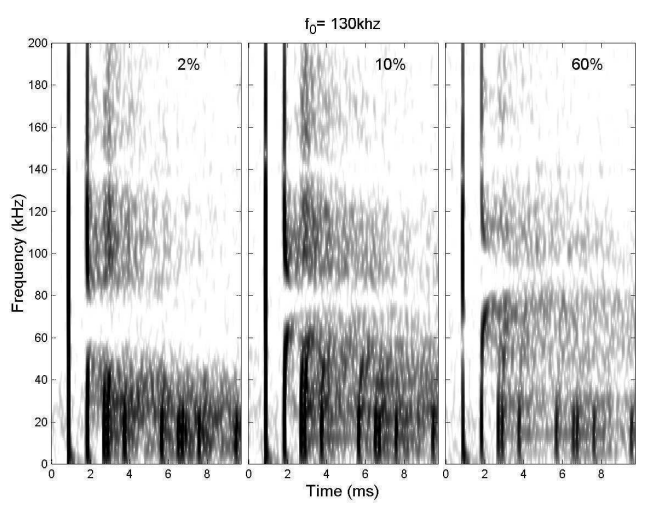

Fig. 3 Fourier spectrograms of magnetostrictive time-history signals for a seven-wire strand of 10 - $\mathrm{m}$ length and $15.9 \mathrm{~mm}$ in diameter excited at $\mathrm{f}_{0}=130 \mathrm{kHz}$ at different tensile load strengths.

\section{Numerical model}

\subsection{The SAFE method}

One assumes a linearly elastic material, small strains and displacements and a time harmonic dependence. There is no external force. The 3D variational formulation governing elastodynamics is given by:

$\int_{\Omega} \delta \boldsymbol{\varepsilon}^{T} \mathbf{C} \boldsymbol{\varepsilon} d V-\omega^{2} \int_{\Omega} \rho \delta \mathbf{u}^{T} \mathbf{u} d V=0$

for any kinematically admissible trial displacement field $\delta \mathbf{u} . \delta \boldsymbol{\varepsilon}$ denotes the virtual strain vector $\left[\begin{array}{llllll}\delta \varepsilon_{11} & \delta \varepsilon_{22} & \delta \varepsilon_{33} & 2 \delta \varepsilon_{12} & 2 \delta \varepsilon_{13} & 2 \delta \varepsilon_{23}\end{array}\right]^{T}$. The superscript $T$ denotes the matrix transpose. Subscripts $i=1,2,3$ are the respective components in the considered coordinate system, denoted $(x, y, s)$ - which might be different from the Cartesian coordinate system denoted $(X, Y, Z)$. C is the matrix of material properties. $\rho$ is the material density and $\Omega$ is the structural volume. In the remainder of this paper, $s$ will denote the waveguide axis (not necessarily straight).

The strain-displacement relation can be written as follows:

$\boldsymbol{\varepsilon}=\left(\mathbf{L}_{x y}+\mathbf{L}_{s} \partial / \partial s\right) \mathbf{u}$ 
where $\mathbf{L}_{x y}$ is the operator containing all terms that do not contain derivatives with respect to the axis $s$. Now we further assume a $e^{i k s}$ dependence, $k$ being the axial wavenumber. The problem is hence reduced from three to two dimensions (from the volume $\Omega$ to the cross-section $S$ of the waveguide). Then, the FE discretisation of Eq.(3.1) finally leads to the following eigenvalue problem:

$\left\{\mathbf{K}_{1}-\omega^{2} \mathbf{M}+i k\left(\mathbf{K}_{2}-\mathbf{K}_{2}^{T}\right)+k^{2} \mathbf{K}_{3}\right\} \mathbf{u}=\mathbf{0}$

with the following elementary matrices:

$$
\begin{gathered}
\mathbf{K}_{1}^{e}=\int_{S^{e}} \mathbf{N}^{e T} \mathbf{L}_{x y}^{T} \mathbf{C L}_{x y} \mathbf{N}^{e} d S, \quad \mathbf{K}_{2}^{e}=\int_{S^{e}} \mathbf{N}^{e T} \mathbf{L}_{x y}^{T} \mathbf{C} \mathbf{L}_{s} \mathbf{N}^{e} d S \\
\mathbf{K}_{3}^{e}=\int_{S^{e}} \mathbf{N}^{e T} \mathbf{L}_{s}^{T} \mathbf{C} \mathbf{L}_{s} \mathbf{N}^{e} d S, \quad \mathbf{M}^{e}=\int_{S^{e}} \rho \mathbf{N}^{e T} \mathbf{N}^{e} d S
\end{gathered}
$$

where $\mathbf{N}^{e}$ is a matrix containing nodal interpolating functions of the element. The solution of Eq. (3) yields the propagation modes. At fixed real $k$, the eigenproblem Eq. (3.3) is linear for finding $\omega^{2}$. This simpler approach is useful only if interest is restricted to propagating modes in undamped systems. Given $\omega$ and finding $k$, the eigenproblem is quadratic. It can be recast into a generalized linear eigensystem written for $\left[\begin{array}{ll}\mathbf{u} & k \mathbf{u}\end{array}\right]^{T}$ in order to be solved by standard numerical solvers - see (Tisseur and Meerbergen 2001) for instance.

\subsection{Helical coordinate system}

In the previous section, we have assumed a $e^{i k s}$ dependence. This is equivalent to perform a spatial Fourier analysis in the $s$ direction. This dependence indeed implies the following assumptions:

- $\quad$ the cross-section of the waveguide does not vary along $s$ (condition 1);

- the material properties do not vary along $s$ (condition 2, which is assumed to be satisfied throughout this work);

- the $(x, y, s)$ coordinate system is so that $s$ does not appear explicitly in the coefficients of the equilibrium equations (condition 3 )

Verifying this last condition (condition 3 ) is somewhat more technical than the first two.

First, the helix centerline curve must be described by the following position vector in the Cartesian orthonormal basis:

$$
\mathbf{R}(s)=R \cos \left(\frac{2 \pi}{l} s\right) \mathbf{e}_{X}+R \sin \left(\frac{2 \pi}{l} s\right) \mathbf{e}_{Y}+\frac{L}{l} s \mathbf{e}_{Z}
$$

where $l=\sqrt{L^{2}+4 \pi^{2} R^{2}} . R$ and $L$ are respectively the radius of the centreline in the $(X, Y)$ Cartesian plane and the helix step along the $Z$ axis. The unit tangent, 
normal and binormal vectors to the centreline are respectively obtained from $\mathbf{T}=d \mathbf{R} / d s$ and the Serret-Frenet formulae, $d \mathbf{T} / d s=\kappa \mathbf{N}$ and $d \mathbf{N} / d s=\tau \mathbf{B}-\boldsymbol{\kappa} \mathbf{T}$. Both curvature $\kappa=4 \pi^{2} R / l^{2}$ and tortuosity $\tau=2 \pi L / l^{2}$ are constant. For clarity, Fig.4 exhibits one helix step and its Serret-Frenet basis.

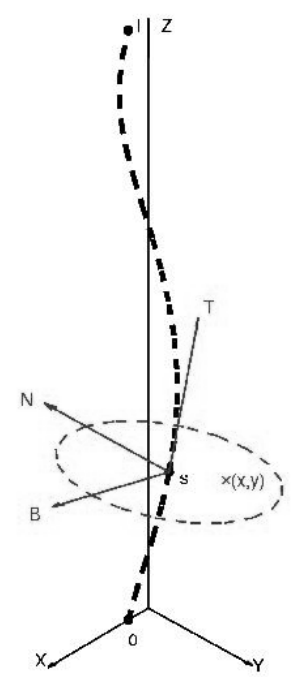

Fig. 4. Helix step and its Serret-Frenet basis with $(x, y)$ the helical cross-section coordinates.

Now, a new coordinate system is constructed from the orthonormal basis $(\mathbf{N}, \mathbf{B}, \mathbf{T})$ as follows:

$\mathbf{X}(x, y, s)=\mathbf{R}(s)+x \mathbf{N}(s)+y \mathbf{B}(s)$

yielding the covariant basis $\left(\mathbf{g}_{1}, \mathbf{g}_{2}, \mathbf{g}_{3}\right)=(\partial \mathbf{X} / \partial x, \partial \mathbf{X} / \partial y, \partial \mathbf{X} / \partial s)$ (nonorthogonal). It could be checked that the metric tensor, defined by $(\mathbf{g})_{i j}=\mathbf{g}_{i} \cdot \mathbf{g}_{j}$, does not depend on the third curvilinear coordinate $s$. One consequence is that the coefficients of the partial differential equilibrium equations are not dependent upon $s$ either (condition 3 is satisfied).

In Eqs. (3.3) and (3.4), covariant components have been chosen for $\boldsymbol{\varepsilon}$ while components with respect to $(\mathbf{N}, \mathbf{B}, \mathbf{T})$ have been preferred for $\mathbf{u}$. Also, the components of $\mathbf{C}$ should be understood as contravariant. Assuming an isotropic material, $\mathbf{C}$ can be obtained from the following tensor expression:

$C^{i j k l}=\frac{v E}{(1+v)(1-2 v)} g^{i j} g^{k l}+\frac{E}{2(1+v)}\left(g^{i k} g^{j l}+g^{i l} g^{j k}\right)$

where $g^{i j}$ is the contravariant metric tensor, $v$ and $E$ denotes Poisson coefficient and Young modulus - for more details, see (Treyssede 2008). 
Now, consider a single helical wire of circular cross-section. The cross-section obviously does not change along the helical axis $s$ (condition 1 is fulfilled) so that the proposed approach is valid. The analysis of a straight wire can also be readily performed, the cylinder corresponding to the special case $\kappa=\tau=0$.

However, a question arises about the choice of the invariant coordinate system to be used when considering helical structures made of both straight and helical wires. Of course, the choice $\kappa=\tau=0$ (resp. $\kappa \neq 0, \tau \neq 0$ ) cannot be applied because condition 1 would not be satisfied for helical (resp. straight) wires.

The adequate system is indeed given by $\kappa=0, \tau=2 \pi / L(R=0)$, corresponding to a rotating coordinate system around the $Z$ axis ( $s \equiv Z$ ). With this system, a central straight wire (cylinder) has an invariant circular cross-section along $Z$ ("a rotating circle remains a circle"). Furthermore, the cross-sections of peripheral helical wires do not change either along $Z$ (but their shape in the $(x, y)$ plane is not circular any more).

\subsection{Numerical results}

The material is assumed to be isotropic, with no material damping. For a steel wire, a typical value of 0.30 will be chosen for the Poisson coefficient. We consider waveguides with a circular cross-section of radius $a$. The adimensionalized angular frequency is given by $\omega a / c_{s}$, with $c_{s}$ the shear velocity. Six-node triangles meshes will be used. FE computations are held at fixed real wavenumbers $k$. As a preliminary result, one single wire is first considered. Both cylindrical $(\kappa=\tau=0)$ and helical geometries $(\kappa \neq 0, \tau \neq 0)$ are analysed. For the helical waveguide, a small lay angle of $\phi=\tan ^{-1}(2 \pi R / L)=7.5^{\circ}$ with $R=2 a$ has been chosen (typical values for civil engineering seven wire strands). Figure 5 exhibits the dispersion curves for both cases. Few differences are observed. For the helical waveguide, flexural modes do not occur in pairs of equal wavenumbers, while wavenumbers of compressional and torsional modes remain nearly unchanged (Treyssede 2007). It could be checked that the rotating system $(\kappa=0, \tau=2 \pi / L)$

yields some physically equivalent results, thus demonstrating its adequacy (not shown in this paper for conciseness).

The wave modes propagating inside a typical seven-wire strand are now studied. Stick contact conditions are assumed for simplicity (no slip, no separation and no friction are considered). $a$ denotes the central wire radius $(a=2.7 \mathrm{~mm})$. Peripheral wires radii are chosen as $0.97 a$, with the same lay angle as before. The coordinate system to be used is $(\kappa a=0, \tau a=0.0668)$. 


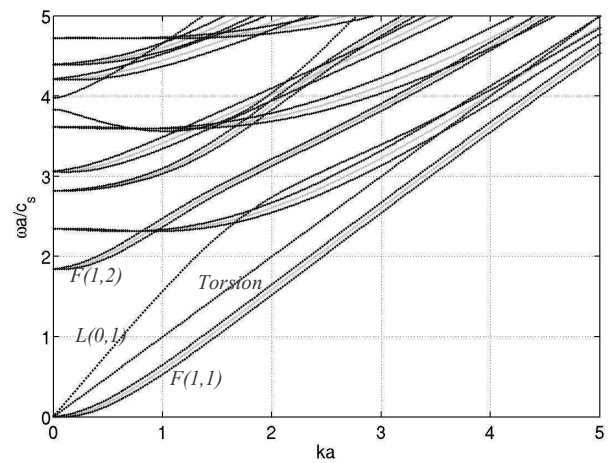

Fig. 5 Plot of frequency versus wavenumber. Black: helical, gray: cylindrical waveguides

The FE mesh, corresponding to a $(X, Y)$ plane cut, is given in Fig. 6.

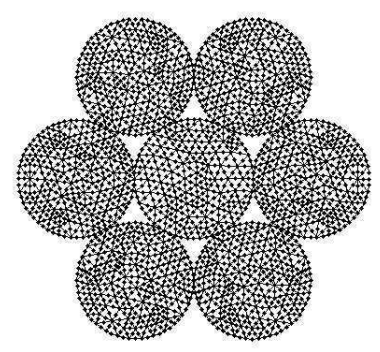

Fig. $6(X, Y)$ plane cross-section FE mesh used for the seven-wire strand $\left(\phi=7.5^{\circ}\right)$

Figure 7 exhibits the energy velocity curves for the adimensional frequency range [0;2]. Single wire curves are also given for comparison (for both central and peripheral wires). Due to a strong inter-wire coupling, a far more complex behaviour is observed for the seven wire strand. The most striking phenomenon is a seemingly cut-off of the fastest mode (compressional-like $L(0,1)$ mode) around $\omega a / c_{s}=0.4$, corresponding to about $75 \mathrm{kHz}$, which is in agreement with experiments where a mean frequency of $70 \mathrm{kHz}$ is deduced from the spectrogram (and associated to group velocities). In fact, this apparent cut-off is due to some strong bifurcations occurring near 0.4 as clearly shown by Fig. 8 .

The upper curve of Fig. 7 is composed of two distinct branches, describing the behaviour of two distinct wave modes. 

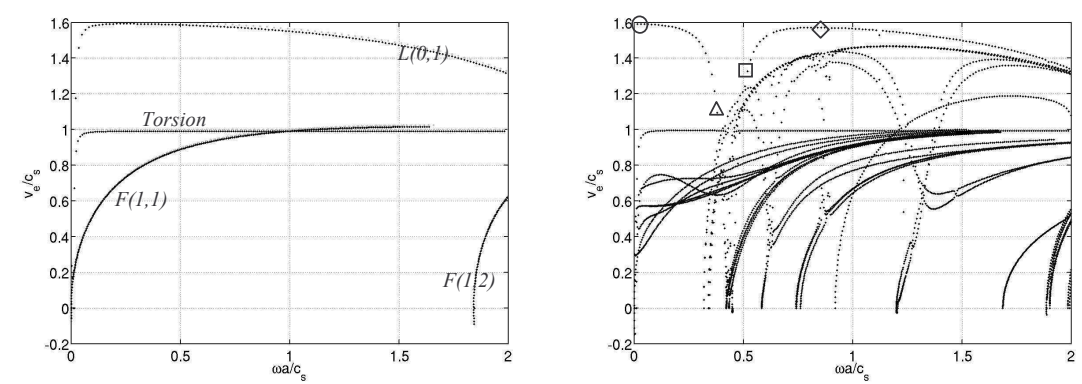

Fig. 7 Plot of energy velocity (a) single wire (black: peripheral, gray: central, (b) seven-wire strand $\left(\phi=7.5^{\circ}\right)$

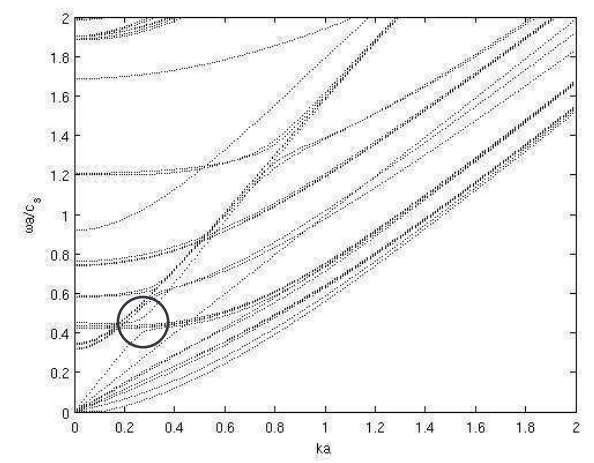

Fig. 8 Plot of frequency vs wavenumber for the seven-wire strand. Circle: bifurcation zone

Finally, Fig. 9 shows modeshapes computed for $\omega a / c_{s}=0.03$ and 0.38 (left branch), $\omega a / c_{s}=0.52$ and 0.81 (right branch).
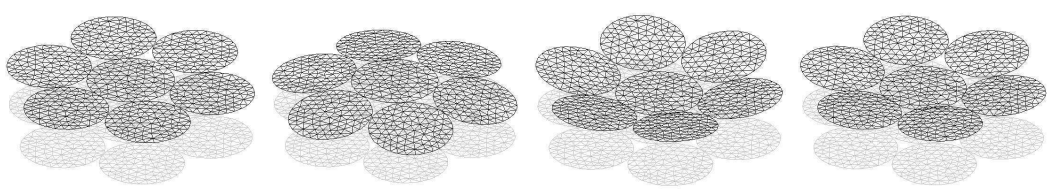

Fig. 9 Modeshapes computed for: $\triangle \omega a / c_{s}=0.03, \triangle 0.38, \square 0.52, \diamond 081$ (symbols of Fig. 7) 


\section{Conclusions}

Elastic wave propagation inside helical wires has been analysed through a SAFE method based on a translationally invariant helical coordinate system. The special case of no curvature yields an adequate coordinate system to be used for the analysis of helical structures made of a straight core and peripheral helical wires. Dispersion inside a typical $6+1$ strand has been investigated by assuming stick contact conditions. A far more complex behaviour than for single wires has been observed. However, it is worth noting, that the fastest compressional-like mode of the seven-wire strand dispersion behaviour looks like the $L(0,1)$ one for a single wire, excepted in a frequency region typically around $75 \mathrm{kHz}$ where an apparent band-cut zone is observed. This intrinsic seven-wire strand low-frequency dispersion behaviour agrees well the experimental results obtained with encircling longitudinally-polarized magnetostrictive transducers on a seven-wire strand. Thus, the proposed seven-wire propagation model allows a novel interpretation, through the strong bifurcation between two distinct modes, of the missing notch frequency experimentally observed here and in the literature. Future works will be devoted to the numerical modelling of the loading effect.

\section{References}

Beard M. D., Lowe M. J. S., Cawley P.: Ultrasonic guided waves for inspection of grouted tendons and bolts. J. Mater. Civil Eng. 212, 212-218 (2003)

Damljanovic V., Weaver R. L.: Propagating and evanescent elastic waves in cylindrical waveguides of arbitrary cross-section. J. Acoust. Soc. Am. 115, 15721581 (2004)

Demma A., Cawley P., Lowe M.: The effect of bends on the propagation of guided waves in pipes. J. Press Vess.-T ASME 127, 328-335 (2005)

Finneveden S., Fraggstedt M.: Waveguide finite element for curved structures. J. Sound Vib. 312, 644-671 (2008)

Gavric L.: Computation of propagative waves in free rail using a finite element technique. J. Sound Vib. 185, 531-543 (1995)

Gry L., Gontier C.: Dynamic modeling of railway track: a periodic model based on a generalized beam formulation. J. Sound Vib. 199, 531-558 (1997)

Hayashi T., Song W.-J., Rose J. L.: Guided wave dispersion curves for a bar with an arbitrary cross-section, a rod and rail example. Ultrasonics 41, 175-183 (2003)

Kwun H., Bartels K. A., Hanley J. J.: Effects of tensile loading on the properties of elastic-wave propagation in a strand. J. Acoust. Soc. Am. 103, 3370-3375 (1998) 
Laguerre L., Brissaud M., Aime J.-C.: Magnetostrictive pulse-echo device for non-destructive evaluation of steel cylindrical materials using guide waves. Ultrasonics 39, 503-514 (2002)

Laguerre L., Brissaud M., Aime J.-C. : Low frequency ultrasound reflectometry device based on magnetoelastic transducers for the non-destructive evaluation of steel rods and cables. Bull. Lab. Ponts et Chaussées 239, 7-27 (2003)

Lanza di Scalea F., Rizzo P., Seible F.: Stress measurement and defect detection in steel strands by guided stress waves. J. Mater Civil Eng. 15, 219-227 (2003)

Mace B. R., Duhamel D., Brennan M. J.: Finite element prediction of wave motion in structural waveguides. J. Acoust Soc. Am. 117, 2835-2843 (2005)

Mead D. J.: A general theory of harmonic wave propagation in linear periodic systems with multiple coupling. J. Sound Vib. 27, 235-260 (1973)

Mead D. J.: Wave propagation in continuous periodic structures: research contributions from Southampton, 1964-1995. J. Sound Vib. 190, 495-524 (1996)

Onipede O., Dong S. B.: Propagating waves and end modes in pretwisted beams. . J. Sound Vib. 195, 313-330 (1996)

Pavlakovic B., Lowe M. J. S., Cawley P.: Inspection of tendons in post-tensioned concrete using guided ultrasonic waves. Insight 41, 446-452 (1999)

Pavlakovic B., Lowe M. J. S., Cawley P.: High-frequency low-loss modes in imbedded bars. J. App. Mech. 68, 67-75 (2001)

Rizzo P., Lanza di Scalea F.: Wave propagation in multi-wire strands by waveletbased laser ultrasound. Exp. Mech. 44, 407-415 (2004)

Tisseur F., Meerbergen K.: The quadratic eigenvalue problem. SIAM Rev. 43, 235-286 (2001)

Treyssede F.: Numerical investigation of elastic modes of propagation in helical waveguides. J. Acoust. Soc. Am. 121, 3398-3408 (2007)

Treyssede F.: Elastic waves in helical waveguides. Wave Motion 45, 457-470 (2008) 